\title{
PLANEJAMENTO ESTRATÉGICO SITUACIONAL APLICADO À ATENÇÃO FARMACÊUTICA: EXPERIÊNCIAS E POSSIBILIDADES*
}

Silvana Teresinha Giacobo

Serviço de Atenção Especializada (SAE), Ambulatório de Dermatologia Sanitária

Secretaria Estadual de Saúde/Rio Grande do Sul, Brasil

Cristiane Barelli

Universidade de Passo Fundo, Faculdade de Medicina

Passo Fundo, Rio Grande do Sul, Brasil (autor para correspondência)

*E-mail: silvana.giacobo@hotmail.com

Carla Beatrice Crivellaro Gonçalves e Siomara Regina Hahn

Universidade de Passo Fundo, Instituto de Ciências Biológicas, Curso de Farmácia

Passo Fundo, Rio Grande do Sul, Brasil

\section{Resumo}

O objetivo deste estudo de caso é relatar e avaliar a construção do plano operativo para implementação da atenção farmacêutica na rede básica de saúde do município de Tapera, Rio Grande do Sul (RS). Dentre as dificuldades vivenciadas pela assistência farmacêutica na unidade de saúde o problema prioritário foi a má adesão ao tratamento medicamentoso por parte dos usuários. A metodologia adotada como proposta de intervenção foi a concepção matusiana do Plano Estratégico Situacional (PES), incluindo os momentos explicativo, normativo, estratégico e tático-operacional. A imagem-objetivo projetada pelos atores que discutiram o PES foi a monitorização adequada dos portadores de doenças crônicas não transmissíveis, com quadros clínicos compensados, fator determinante para o aumento da expectativa e da qualidade de vida, além da diminuição de agravos e de custos para o sistema de saúde. A integração do profissional farmacêutico na rotina de atendimento aos hipertensos e diabéticos após a implementação do PES, por meio da orientação farmacêutica, resultou no desenvolvimento de ações conjuntas, com a equipe de saúde da família, otimizando a adesão ao tratamento farmacológico e promovendo o uso racional de medicamentos.

Palavras-chave: Assistência farmacêutica, gestão em saúde, planejamento estratégico situacional, atenção farmacêutica, doenças crônicas não transmissíveis.

\section{Strategic planning situational applied to pharmaceutical care: experiences and possibilities}

\section{Abstract}

The objective of this case study is to describe and evaluate the construction of the operational plan for implementation of pharmaceutical care in primary health care in the city of Tapera, Rio Grande do Sul. Among the difficulties experienced by the pharmaceutical care at the clinic the priority problem was poor adherence to drug treatment by the patients. The methodology adopted as an intervention proposal was matusiana design of the Situational Strategic Plan (PES), including explanatory, normative, strategic and tactical-operational times the target image projected by the actors who discussed the PES was the appropriate monitoring of patients with diseases non-communicable diseases chronic, compensated with medical conditions, determining factor for the increase in expectation and quality of life, beyond the reduction of harm and costs to the health system. The integration of pharmacists in routine care for hypertension and diabetes after the implementation of PES, through 
pharmaceutical orientation has resulted in the development of joint actions with the family health team, optimizing adherence to pharmacological treatment and promoting the use rational drug.

Keywords: Pharmaceutical services, health management, Situational strategic planning, pharmaceutical care, chronic diseases.

\section{Planificación estratégica situacional aplicada a la atención farmacéutica: experiencias y posibilidades}

\section{Resumen}

El objetivo de este estudio de caso es describir y evaluar la construcción del plan operativo para la implementación de la atención farmacéutica en la red primaria de salud del municipio de Tapera, Río Grande do Sur (RS). De entre las dificultades experimentadas por la atención farmacéutica en la clínica el problema prioritario era mala adherencia al tratamiento farmacológico por los usuarios. La metodología adoptada como una propuesta de intervención fue la concepción matusiana del Plan Estratégico Situacional(PES), incluyendo los momentos explicativos, normativos, estratégicos y táctico-operacional. La imagen-objetivo proyectada por los actores que discutieron el PES fue el monitorizado adecuadamente de los pacientes con enfermedades crónicas no transmisibles, con cuadros clínicos compensados, factor determinante para el aumento de la expectativa y de la calidad de vida, además de la reducción de los daños y de los costos para el sistema de salud. La integración de los farmacéuticos en la atención de rutina para la hipertensión y la diabetes después de la implementación del PES, a través de la orientación farmacéutica, se ha traducido en el desarrollo de acciones conjuntas, con el equipo de salud de la familia, la optimización de la adherencia al tratamiento farmacológico y la promoción del uso racional de los medicamentos.

Palabras clave: Asistencia farmacéutica, gestión en salud, planificación estratégica situacional, atención farmacéutica, enfermedades crónicas no transmisibles.

\section{INTRODUÇÃO}

A atenção farmacêutica, como prática desenvolvida no âmbito da assistência farmacêutica, pode ser considerada no Brasil como uma atividade recente que desperta interesse e se incorpora gradualmente nas ações dos farmacêuticos no país(1).

A concepção dessa prática tem origem no trabalho de farmacêuticos hospitalares nos Estados Unidos, na década de 70, que passam a atuar clinicamente na terapêutica do paciente. Entretanto, foi somente em 1990 que os americanos Hepler e Strand ${ }^{(2)}$ iniciam a publicação de vários trabalhos apontando para uma nova atribuição do profissional farmacêutico. Esses autores defendem "a adoção de um enfoque centrado no paciente e o desenvolvimento de uma relação terapêutica na qual o paciente e o profissional trabalhem juntos para resolver os problemas relacionados aos medicamentos". Também orientam que seja assumida a responsabilidade sanitária de prevenir a morbidade com os medicamentos, incluindo as atividades relacionadas ao seu uso seguro e racional.

A construção do conceito de atenção farmacêutica adotada pela Organização Mundial da Saúde (OMS), tal como o conhecemos hoje, converge com a concepção americana e foi divulgada por meio da Declaração de Tóquio em 1993. Portanto, para a Organização Panamericana de Saúde e Organização Mundial da Saúde (Opas-OMS)(3), a atenção farmacêutica é definida como "um compêndio das atitudes, dos comportamentos, dos compromissos, das inquietações, dos valores éticos, das funções, 
dos conhecimentos, das responsabilidades e das destrezas do farmacêutico na prestação da farmacoterapia, com o objetivo de alcançar resultados terapêuticos definidos na saúde e na qualidade de vida do paciente".

Em 2001, ao formular as diretrizes para o desenvolvimento da atenção farmacêutica, os espanhóis ampliam esse conceito incluindo as atividades tradicionais no novo modelo. Assim, a dispensação, a indicação de medicamentos que não necessitam de prescrição, a educação sanitária, a farmacovigilância, o segmento farmacoterapêutico e todas as atividades que se relacionam com o uso racional de medicamentos passam a integrar as ações do denominado Consenso Espanhol para a Atenção Farmacêutica(4).

No Brasil, a concepção filosófica, que no decorrer das duas últimas décadas norteou a construção Sistema Único de Saúde (SUS), como sistema universal, integral, equânime, de qualidade, e com participação popular, permeia também as definições de Assistência Farmacêutica. Os documentos intitulados Política Nacional de Medicamentos e Política Nacional da Assistência Farmacêutica, publicados respectivamente em 1998 e 2004, contemplam diretrizes amplas que abordam desde a pesquisa e produção de fármacos até a garantia de utilização racional e segura dos medicamentos pelos usuários ${ }^{(5-6)}$.

O Consenso Brasileiro de Atenção Farmacêutica foi proposto em 2002 e, como no modelo espanhol, as atividades relacionadas ao paciente foram consideradas e estabelecidas no que se denominou de macrocomponentes, quais sejam: "educação em saúde, orientação farmacêutica, dispensação, atendimento farmacêutico, acompanhamento/ seguimento farmacoterapêutico, registro sistemático das atividades e mensuração e avaliação dos resultados"(7). Aproximando-se dos princípios do SUS, o consenso brasileiro inclui como atividade profissional do farmacêutico; compromissos e responsabilidades com a prevenção de doenças, a promoção e recuperação da saúde de forma integrada à equipe de saúde.

Desde então, as principais experiências de incorporação das atividades de atenção farmacêutica na atuação rotineira desse profissional estão vinculadas às doenças crônicas não transmissíveis (DCNTs), especialmente a hipertensão arterial e diabetes ${ }^{(8-13)}$.

Em Goiânia, Goiás, foi realizado o acompanhamento de 50 pacientes durante 12 meses no âmbito da Estratégia de Saúde da Família(9). Desses, $80 \%$ apresentavam mais de uma patologia associada, com predomínio de hipertensão arterial sistêmica, e 92\% utilizavam dois ou mais fármacos simultaneamente. Os autores detectaram 154 Problemas Relacionados com Medicamentos (PRM), com incidência de 3,1 PRM por paciente, sendo o mais frequente a falta de efetividade na terapêutica (49\%), sendo $26,3 \%$ desses devido à falta de adesão ao tratamento. O diagnóstico realizado revelou a necessidade de seguimento farmacoterapêutico para os pacientes portadores de doenças crônicas.

Castro et al. ${ }^{(8)}$ descrevem a experiência de seguimento farmacoterapêutico realizada por farmacêuticos no Ambulatório de Cardiologia do Hospital de Clínicas de Porto Alegre, Rio Grande do Sul, desde 2001, na qual evidenciam o sucesso na diminuição da pressão arterial de pacientes hipertensos não controlados.

Um estudo realizado no Paraná(12) com pacientes portadores de diabetes tipo 2 demonstrou que a consulta farmacêutica realizada a cada quadrimestre foi capaz de melhorar os parâmetros bioquímicos dos pacientes e minimizar fatores de risco importantes para agravos relacionados à complicações diabéticas. Os pesquisadores identificaram reduções na glicemia $(P<0,0001)$, hemoglobina glicada $(P=0,0022)$, colesterolemia $(P=0,0072)$ e pressão arterial $(P<0,0001)$.

Logo, essas patologias são eleitas para intervenções de saúde por critérios de elevada prevalência. Além de demandarem acompanhamento sistemático da equipe de saúde e do tratamento, baseiam-se em medidas farmacológicas e não farmacológicas, passíveis de intervenção farmacêutica. As medidas não farmacológicas, em geral, estão ligadas a mudanças no estilo de vida e as farmacológicas, ao uso regular e contínuo de um ou mais fármacos, pressupondo a adesão ao tratamento. A função desses fármacos, em geral, é o controle dos sintomas e a prevenção de agravos (prevenção terciária e quaternária)(3,8-13).

Nesse contexto, as dificuldades de adesão ao tratamento aparecem como um problema que merece especial atenção das equipes de saúde e um desafio para o cuidado integral e resolutivo em saúde. 
Segundo a OMS (14), o grau de coincidência entre a orientação médica/equipe de saúde e o comportamento do paciente é considerado adesão ao tratamento. Outros autores ${ }^{(17-18)}$ definem adesão como a habilidade do paciente em desenvolver um papel ativo frente ao seu tratamento. Porém, a Organização Mundial da Saúde considera que a adesão ao tratamento não se resume ao comportamento do paciente frente a uma prescrição; é multifatorial e incluem aspectos referentes ao sistema de saúde, fatores socioeconômicos, além de aspectos relacionados ao tratamento, ao paciente e à própria doença ${ }^{(14)}$.

A compreensão de que adesão a um tratamento é multicausal foi a premissa do planejamento estratégico situacional desenvolvido neste trabalho, propiciando o desenvolvimento das ações de Atenção Farmacêutica na Unidade Básica de Saúde Centro, do município de Tapera, no Estado do Rio Grande do Sul.

O objetivo desse estudo de caso é relatar o processo de implementação da atenção farmacêutica no município de Tapera, RS, a partir da aplicação do Planejamento Estratégico Situacional. Também foram identificadas as fragilidades e potencialidades decorrentes do processo participativo e interativo entre todos os atores envolvidos no serviço, a negociação e pactuação de ações e responsabilidades com base na análise do contexto local.

\section{METODOLOGIA}

O trabalho é resultante do desenvolvimento de um plano operativo de intervenção na rede municipal de saúde de Tapera, RS, pelo Curso de Especialização em Gestão da Assistência Farmacêutica do Ministério da Saúde ${ }^{(19)}$. Trata-se de um estudo de caso fundamentado por meio da pesquisa avaliativa em saúde, com caráter observacional e enfoque quantitativo e qualitativo ${ }^{(20-21)}$. Foi realizado de janeiro a setembro de 2012.

O plano operativo desenvolvido nesse estudo de caso foi elaborado a partir da concepção do Planejamento Estratégico Situacional (PES) ${ }^{(22-23)}$. Esse método representa uma das três vertentes do planejamento estratégico latino-americano que faz a ruptura com o planejamento normativo tradicional. Considera o ato de planejar como a ciência ou a arte de construir maior governabilidade a partir da análise dos elementos em cena. Atores, situações e ações formam um complexo que, partindo do diagnóstico da realidade, busca a construção de um cenário ideal, definido como imagem-objetivo(22).

O processo de planificação situacional definido por Matus é composto por quatro elementos ${ }^{(20)}$ :

a) Momento explicativo, que se refere à construção de explicações para fundamentar a própria ação e interferir e compreender a ação dos componentes;

b) Momento normativo, que se refere à seleção das operações e ações necessárias para atingir os objetivos;

c) Momento estratégico que se refere a explorar diferentes modos de jogar, considerando os oponentes e os aliados, para incorporar viabilidade ao plano;

d) Momento tático-operacional que é o momento da ação. Esta, porém, nunca é a mera execução do plano, mas uma adaptação deste às circunstâncias do momento.

Embasados nesta concepção teórica, a construção do plano operativo, tema deste estudo, teve como preocupação inicial o desenvolvimento de um trabalho que fosse capaz de identificar as lacunas na assistência farmacêutica no local da pesquisa e propor ações organizadas para o seu enfrentamento. 


\subsection{Caracterização do local do estudo}

O município de Tapera está localizado na região norte do Rio Grande do Sul, a 280 quilômetros da capital Porto Alegre. Tem uma população estimada para 2014 de 10.796 habitantes e 95,9\% de cobertura na Estratégia de Saúde da Família (ESF) desenvolvida por quatro equipes ${ }^{(24)}$.

O local definido para o estudo de caso foi a farmácia vinculada à Unidade Básica de Saúde Centro. Nesse espaço, além de uma equipe de ESF, estão abrigados o Pronto Atendimento Municipal, algumas especialidades médicas, o laboratório de análises clínicas, o setor de fisioterapia, fonoaudiologia, assistência social, a equipe de saúde mental e a estrutura administrativa da Secretaria Municipal de Saúde.

Na ocasião do desenvolvimento do estudo de caso, a organização dos serviços municipais de saúde, além da farmácia UBS Centro, contava com duas farmácias públicas com dispensação de medicamentos da lista básica. A dispensação dos psicotrópicos e dos medicamentos dos componentes especiais, especializados e estratégicos ocorria centralizada na UBS Centro.

A farmácia central, além da atividade de dispensação, é responsável por todas as etapas da assitência farmacêutica: seleção, programação, encaminhamento para aquisição, recebimento, armazenamento e distribuição para as outras unidades de saúde.

Algumas ações no âmbito da atenção farmacêutica eram realizadas pela farmacêutica local, com destaque para a orientação farmacêutica e ações de educação em saúde desenvolvidas junto a alguns grupos de usuários da rede pública. Percebe-se, entretanto, persistência de problemas de baixa adesão ao tratamento farmacológico por uma importante parcela de usuários de medicamentos na forma crônica, assistidos por este serviço.

Esse setor foi eleito para o desenvolvimento do plano operativo pelas seguintes razões: é o local de atuação da farmacêutica, no qual a profissional possui maior governabilidade, concentra o maior número de atendimentos farmacêuticos do município, está politicamente e geograficamente próximo aos gestores da Secretaria Municipal, e possui a melhor estrutura física e de recursos para o desenvolvimento do plano. Além disso, dentre os locais de dispensação de medicamentos vinculados à rede pública de saúde, a Farmácia Centro é o local que possui relação direta com o maior número de prescritores.

Quanto aos aspectos éticos este estudo de caso seguiu integralmente as normas e diretrizes regulamentadas pelo Conselho Nacional de Saúde por meio da Resolução N¹96/1996(25).

\section{RESULTADOS E DISCUSSÃO}

Ao considerar que gestão é um processo técnico, político e social capaz de produzir resultados, apostou-se na construção de um plano cuja operacionalização fosse capaz de produzir impacto epidemiológico e sanitário no município ${ }^{(26-27)}$.

Aumentar a qualidade e a expectativa de vida dos portadores de doenças crônicas não transmissíveis, por meio de ações articuladas com toda a equipe da saúde da unidade básica, utilizando apenas os recursos disponíveis, foi o desafio proposto para a produção de resultados.

O processo político e social do PES pôde ser vivenciado na condução do processo de mobilização dos atores envolvidos, motivados pela possibilidade de rearranjos nas rotinas rumo ao aumento da qualidade da assistência à saúde.

Dados semelhantes foram observados por Santana e colaboradores ao descrever a experiência de utilização do PES para superação de dificuldades na seleção de medicamentos em hospitais públicos de Sergipe ${ }^{(27)}$. 


\subsection{Momento explicativo}

O momento explicativo visa primordialmente realizar a apreciação da situação que, para Matus, "conduz aos objetivos, e os objetivos à seleção de problemas e ao aprofundamento da explicação" (23).

Assim, para realizar o diagnóstico situacional da assistência farmacêutica foi proposta a realização de uma oficina com a participação da equipe de saúde da unidade básica. Nessa reunião, deveriam ser elencados e priorizados os problemas vinculados ao escopo do plano operativo. Otimizando os recursos disponíveis, optou-se por realizar a oficina na reunião ordinária semanal da equipe da UBS Centro, espaço considerado adequado, uma vez que o mesmo concentra os atores importantes para a gestão da unidade de saúde. Além disso, as agendas e as rotinas do serviço estão estruturadas para esta atividade, há uma cultura de discussão dos problemas neste fórum e a maioria dos atores importantes para o desenvolvimento da oficina geralmente resistem em participar de reuniões extras.

A proposta da reunião foi exposta à chefia da unidade e à direção da Secretaria, tendo como meta também sensibilizar e buscar o apoio desses atores estratégicos, não só para a oficina em si, mas para a avaliação do serviço de farmácia do município e a construção de um plano operativo a partir da ótica do planejamento estratégico situacional.

Procedeu-se, então, o contato individual com cada um dos participantes, explanando a metodologia e objetivos da oficina. Nesse momento vários atores convidados colaboram com sugestões. Leite e Guimarães ${ }^{(19)}$ afirmam que a gestão é um processo dinâmico e contínuo de interação entre distintos saberes, recursos e pessoas, com díspares ideologias e compreensões de mundo e das necessidades que devem ser priorizadas. Logo, as sugestões propostas foram valorizadas e incorporadas à dinâmica da oficina.

Foi elaborado um material audiovisual com os principais elementos de PES e sua importância nos serviços de saúde, contextualizando-as à realidade local. O conceito de cada um dos parâmetros utilizados na matriz de priorização dos problemas mereceu destaque no material elaborado.

A primeira oficina foi realizada em janeiro de 2011, com a presença dos seguintes profissionais: diretora de saúde da Secretaria, equipe da ESF (médico, enfermeira e odontóloga), dois psicólogos, três técnicos de enfermagem e dois atendentes de farmácia. Por se tratar de um período de férias, a participação de outros profissionais ficou comprometida, tais como da assistente social, fonoaudióloga, fisioterapeuta, secretária e da técnica em higiene bucal.

A natureza das intervenções realizadas pelos participantes da oficina após a explanação inicial foi diversificada, sendo relativa às concepções de saúde, à importância do planejamento, à realidade local vinculada aos conceitos acadêmicos apresentados e à interface da assistência farmacêutica com os setores/áreas presentes na reunião, em especial a saúde mental.

A discussão entre os participantes abrangeu desde as características e perfis dos usuários da UBS até os aspectos administrativos e gerenciais da unidade de saúde. Consideramos relevante a importância dada por vários atores ao planejamento em saúde.

A partir da compilação dos relatos, foi organizada a matriz de priorização com treze problemas elencados. Esta foi apresentada aos participantes que, um a um, atribuíram valor a cada problema, justificando-os. Considerando os critérios magnitude, transcendência, vulnerabilidade, urgência e factibilidade elencou-se como prioritário o problema de falta de adesão ou descontinuidade de tratamento, principalmente por usuários de medicamentos de tratamento crônico.

A partir da escolha do problema, o entendimento foi que de as alianças deveriam ser construídas com os profissionais da área clínica (médicos, enfermeiros e técnicos de enfermagem), tanto da ESF como do Pronto Atendimento, já que esses possuem interesse na solução do problema eleito. A definição dos descritores e a organização do diagrama de causas e consequências "espinha de peixe" - passaram a ser organizados com a colaboração da enfermeira da unidade de saúde (figura 1).

Os descritores foram definidos a partir dos processos de trabalho da equipe de saúde que atuava no pronto atendimento. Nesse local, frequentemente são atendidos usuários com crises hipertensivas, angina, sintomas iniciais de enfarto ou acidente 
vascular encefálico, em hipo ou hiperglicemia, em crise asmática ou surto psicótico, agravos possivelmente decorrentes do manejo inadequado das doenças crônicas.

O relato de usuários que não usam corretamente os medicamentos também serviu de base para um dos descritores, bem como a contagem de comprimidos restantes na sacola de medicamentos, confrontando com a posologia prescrita e com a data da última dispensação. A imagem-objetivo foi elaborada a partir do entendimento de qual seria a situação ideal para os usuários de medicamentos contínuos relacionados ao manejo de doença crônica.

A proposta da espinha de peixe (figura 1) foi apresentada em nova reunião de equipe para contribuições e aprovação do grupo legitimando essa etapa do PES.

O fato de visualizar o problema em uma imagem organizada com causas, consequências e cenário ideal, foi considerado fundamental para o convencimento e a sensibilização dos atores em direção à construção da imagem-objetivo. Houve manifestações quanto a possíveis ações mitigadoras que foram acolhidas e, na medida do possível, incorporadas ao planejamento da implementação da atenção farmacêutica no município.

Merece destaque a participação, na segunda oficina, de uma médica com experiência em Saúde da Família, que manifestou comprometimento para o alcance do cenário proposto como imagem-objetivo.

O resultado dos trabalhos realizados durante as duas etapas da oficina foram apresentados à direção da Secretaria Municipal que registrou apoio para o enfretamento do problema priorizado, manifestando concordância com a implementação da atenção farmacêutica no serviço.

A partir dessas definições, a elaboração das matrizes dos momentos normativo estratégico e tático-operacional passou a ocorrer de forma concomitante à execução de várias ações que viabilizaram as condições objetivas para o início da implementação da atenção farmacêutica na unidade de saúde. Assim, foi disparado um processo dinâmico no qual a execução e o planejamento ocorreram no mesmo tempo e espaço, em ritmos variáveis.

\subsection{Momento normativo}

Nas etapas do PES, esse momento se refere à seleção das operações e ações necessárias para atingir os objetivos. O plano adquire a forma de propostas de decisão que devem ser tomadas a partir da situação inicial(19,22-23,27).

O momento normativo inicia com a definição do objetivo geral do plano operativo estabelecido a partir da causa e consequência convergentes do problema priorizado. Nesse estudo de caso, o objetivo foi implantar a atenção farmacêutica no município visando aumentar a qualidade e a expectativa de vida dos portadores de doenças crônicas não transmissíveis (DCNT).

Os objetivos específicos foram relacionados a partir das causas e consequências definidas na espinha de peixe, divididos em três blocos: garantia de acesso aos medicamentos; estabelecimentos de rotinas e desenvolvimento de capacitações/formações para identificar usuários com dificuldades de adesão ao tratamento e implementação da atenção farmacêutica propriamente dita, por meio da oferta de orientação farmacêutica, seguimento farmacoterapêutico e intervenções educativas em saúde (individuais e/ou coletivas).

Procedeu-se a estruturação da matriz do momento normativo com a descrição das operações e ações. É importante registrar que algumas operações passam a ser implementadas concomitantemente à sua elaboração, uma vez que o cenário possibilitava a testagem de sua factibilidade e vulnerabilidade. 


\subsection{Momento estratégico}

Estabelecidos os objetivos, suas operações e ações, partiu-se para a análise de viabilidade e factibilidade. Assim, foram avaliadas, a governabilidade, a partir da estrutura de poder e apoio dos gestores, e a existência ou a possibilidade de obtenção de recursos de todas as ordens para e execução do plano.

Nessa fase, consolidou-se a percepção de que o maior investimento necessário para alcançar os objetivos propostos seria garantir o comprometimento dos trabalhadores de saúde envolvidos no processo.

Investir na força dos argumentos de que uma nova abordagem, denominada atenção farmacêutica, pode contribuir para a melhor adesão dos portadores de doenças crônicas ao tratamento farmacológico foi a estratégia utilizada para sensibilização dos gestores, médicos, enfermeiros, técnicos de enfermagem, auxiliares de farmácia e agentes comunitários de saúde.

Nessa fase ficou evidente que, se por um lado os recursos financeiros necessários para a operacionalização do plano são mínimos, por outro os recursos de tempo, dedicação e parceria entre os colegas de equipe são determinantes para o sucesso do trabalho. Nesse contexto, ações de educação permanente, elaborar e implantar rotinas, com a participação da chefia da unidade de saúde, foram consideradas ações imprescindíveis para assegurar a implementação do plano operativo. Dessa forma, mobilizar recursos humanos a partir de argumentos consistentes foi o maior investimento realizado no momento estratégico.

\subsection{Momento tático-operacional}

A última fase da estruturação do plano operativo foi dedicada à definição das responsabilidades individuais dos atores envolvidos, dos recursos financeiros necessários, dos prazos de execução e da construção dos indicadores de monitoramento e avaliação das ações e operações propostas.

A construção da matriz final foi facilitada pelo fato de que uma parte considerável já estava pactuada e várias operações estavam estruturadas. Encontrava-se em andamento as atividades propostas nos objetivos específicos, referentes à sensibilização e capacitação dos profissionais de nível médio, de realização de intervenções educativas em saúde, de orientação farmacêutica, além do estabelecimento de referência e contrarreferência com os prescritores da unidade de saúde.

Nesse momento foi necessário investir nas atividades que remetiam à manutenção das rotinas implantadas e consolidação do processo, bem como nos procedimentos necessários à avaliação do trabalho em andamento.

Em relação ao processo de avaliação do plano operativo, entende-se que os indicadores capazes de medir o impacto merecem atenção especial. Em última instância são esses indicadores que possuem a capacidade de mensurar o quanto nos aproximamos da imagem-objetivo proposta, nesse caso usuários com patologias crônicas compensadas, com adesão ao tratamento, fazendo uso regular e correto dos medicamentos.

No Rio de Janeiro (RJ) foi avaliado o uso racional de medicamentos (URM) em 547 portadores de hipertensão arterial e diabetes atendidos na atenção primária. Os aspectos do URM ligados à adesão mostraram-se associados de forma estatisticamente significativa com indivíduos hipertensos, brancos, com maior continuidade do cuidado, que trabalhavam e que recebiam recomendações sobre dieta e exercício físico de seus médicos. Nos pacientes diabéticos a adesão foi menor, e em ambos, ficou evidente a importância da orientação da equipe de saúde, mesmo que não tenha sido verificado se havia ou não farmacêutico ${ }^{(14)}$.

Zubioli e colaboradores avaliaram um programa de consulta farmacêutica implementado para pacientes portadores de diabetes tipo 2 em Maringá, São Paulo. Os parâmetros foram monitorados para 50 pacientes, a cada quatro meses, durante um ano e consideraram o estilo de vida, farmacoterapia, exames laboratoriais, sinais vitais e medidas antropométricas. Os resultados evidenciaram a eficiência do programa na melhora da saúde dos pacientes ao reduzir fatores de risco de complicações diabéticas ${ }^{(12)}$. 
Alguns estudos realizados em Goiânia evidenciaram que a atenção farmacêutica, desenvolvida no âmbito da estratégia de saúde da família, representa uma alternativa eficaz na obtenção de melhores resultados clínicos e econômicos melhorando a qualidade de vida dos usuários do SUS ${ }^{(9,12)}$.

Naves e Silver avaliaram 15 centros de saúde no Distrito Federal e verificaram dificuldade de acesso aos medicamentos essenciais, porém o mais grave e que compromete as ações de assistência farmacêutica foram os baixos níveis de compreensão dos pacientes sobre a prescrição $(18,7 \%)$, cenário que reitera a necessidade de implementação da atenção farmacêutica nesses serviços ${ }^{(28)}$.

Para outras doenças crônicas e/ou degenerativas também há relatos da eficácia alcançada com consultas farmacêuticas: assistência aos idosos com doença de Chagas, no Ceará(29) e seguimento de pacientes portadores de mal de Parkinson realizado em Santa Catarina ${ }^{(30)}$

Castro e colaboradores( ${ }^{(8)}$ desenvolveram um ensaio clínico randomizado em 2001, no Ambulatório de Hipertensão do Serviço de Cardiologia do Hospital de Clínicas de Porto Alegre, com objetivo de testar a eficácia de intervenções realizadas por farmacêuticos. Este estudo acompanhou 71 pacientes hipertensos por um período de seis meses e concluiu que os serviços farmacêuticos são exequíveis e contribuem para a diminuição da pressão arterial de pacientes hipertensos não controlados.

Corroborando com as evidências descritas, a implementação da atenção farmacêutica no local estudado visa causar impacto no serviço de saúde por meio da promoção da integralidade, diminuição da demanda e custos associados aos agravos e complicações de pacientes portadores de DCNT, qualificação do cuidado e melhora da expectativa e qualidade de vida dos usuários e suas famílias. Vislumbra-se, ainda, a possibilidade de replicar a experiência do desenvolvimento do PES para outros setores/ áreas da unidade de saúde e na gestão local. Para alguns profissionais da equipe de saúde a construção deste plano operativo proporcionou o primeiro contato com o planejamento estratégico situacional motivando reflexões sobre a prática cotidiana do fazer saúde, tanto no aspecto técnico como humano.

\section{CONSIDERAÇÕES FINAIS}

A construção deste plano operativo foi marcada pelo desafio de aliar uma tarefa acadêmica à necessidade de dar respostas efetivas a problemas vivenciados no cotidiano da assistência farmacêutica de um município gaúcho de pequeno porte.

A formação tecnicista que marca a presente geração dos profissionais da saúde, aliada à rotina intensa de atendimento à demanda, geralmente reprimida, imprime um ritmo de trabalho no qual o planejamento e as técnicas de gestão e avaliação não encontram espaço para a sua efetivação.

Ao propor a ordenação e priorização dos problemas existentes na assistência farmacêutica do município, mais especificamente na farmácia da UBS Centro, a partir da metodologia proposta pelo planejamento estratégico situacional, podese vislumbrar uma nova relação com as questões/problemas do cotidiano.

A percepção da real dimensão dessas questões, em especial do problema priorizado, expressas no diagrama de causas e consequências foi fator determinante para mobilizar a equipe de saúde em direção ao enfrentamento do problema de adesão ao tratamento farmacológico por usuários de medicamentos nas DCNT. As possibilidades de ganhos reais que esse enfretamento poderia trazer, não só para o serviço e os profissionais, mas, principalmente, para os usuários, foi o elemento facilitador de sua operacionalização.

A compreensão de que a mudança de uma dada realidade é mediada por uma complexidade de fatores, entre os quais a capacidade de fazer alianças, se constituiu em fator determinante para o desenvolvimento das ações que possibilitaram o início do processo de construção da imagem-objetivo almejada, desvelando o papel político-gerencial do farmacêutico. 
A construção dessa aliança foi favorecida pela natureza do problema, uma vez que as consequências da não adesão ao tratamento atingem não só a equipe de profissionais da assistência farmacêutica, mas os médicos, a equipe de enfermagem, os agentes comunitários de saúde e a própria direção técnica e administrativa da Secretaria de Saúde.

A não adesão ao tratamento pode descompensar pacientes portadores de DCNT, com possibilidade de ocorrência de agravos. Em geral, tais doenças evoluem para quadros que remetem a intervenções de maior complexidade, com maior investimento de trabalho, de recursos e desgaste dos profissionais, em especial os que atuam no pronto atendimento. Acrescenta-se ainda a essa situação a frustração da própria equipe da saúde da família em não controlar agravos relacionados a causas evitáveis.

A partir desse contexto, a proposição de implementar a atenção farmacêutica como estratégia para a promoção de adesão ao tratamento farmacológico a partir da ferramenta do PES ganha respaldo e possibilidade de ser operacionalizada. Assim, o cenário constituído, inaugura uma nova interlocução entre a assistência farmacêutica e o corpo clínico da unidade de saúde, evidenciando a interface das mesmas e possibilidades de ações conjuntas no cuidado do usuário.

Apropriar-se de conceitos extraídos do PES e aplicá-los na gestão da assistência farmacêutica de um pequeno município, transformou-se em exercício de infinitas possibilidades de aprendizado, realização profissional e motivação para novas e prazerosas descobertas.

\section{REFERÊNCIAS}

1. Andrade TU et al. Scientific production in pharmaceutical care: comparison between Brazil, USA and Spain. Braz. J. Pharm. Sci. [online]. 2013;49(1): 39-47.

2. Hepler CD, Strand LM. Opportunities and responsibilities in pharmaceutical care. Am J Hosp Pharm. 1990;47: 533-384.

3. Organización Pan-Americana de La Salud (Opas-OMS). El papel del famacêutico en la atención a salud: informe de reunión de La OMS, Tokio, Japon,31 ago al 3 sep 1993. Buenas Prácticas de Farmácia: Normas de Calidad de los Servicios Farmacéuticos. La Declaración de Tokio - Federación Internacional Farmacéutica. Washington: PAHO;1995.

4. Angonesi D, Sevalho G. Atenção Farmacêutica: fundamentação conceitual e crítica para um modelo brasileiro. Rev. Ciência \& Saúde Coletiva. 2010;15 (Suppl.3): 3603.

5. Brasil. Ministério da Saúde. Secretaria de Ciência, Tecnologia e Insumos Estratégicos. Departamento de Assistência Farmacêutica e Insumos Estratégicos. Diretrizes para estruturação de farmácias no âmbito do Sistema Único de Saúde. Brasilia: Ministério da Saúde, 2009.

6. Marin N, editor. Assistência farmacêutica para gerentes municipais. Rio de Janeiro: OPAS/OMS, 2003.

7. Ivama AM et al. Consenso brasileiro de atenção farmacêutica: proposta. Brasília: Organização Pan-Americana da Saúde, 2002.

8. Castro MS et al. Contribuição da atenção farmacêutica no tratamento de pacientes hipertensos. Rev Bras Hipertens. 2006;13(13): 198-202.

9. Provin MP et al. Atenção Farmacêutica em Goiânia: Inserção do farmacêutico na Estratégia Saúde da Família. Saúde Soc 2010;19(3): 717-723. 
10. Pinheiro RM. Serviços farmacêuticos na atenção primária em saúde. Rev Tempus Actas Saúde Colet. 2010;4(3): 15-22.

11. Martins BPR et al. Pharmaceutical Care for hypertensive patients provided within the Family Health Strategy in Goiânia, Goiás, Brazil. Braz. J. Pharm. Sci. 2013;49(3): 609-618.

12. Zubioli A et al. Pharmaceutical consultation as a tool to improve health outcomes for patients with type 2 diabetes. Braz. J. Pharm. Sci. 2013;49(1): 85-94.

13. Mendes LVP, Luiza VL, Campos MR. Uso racional de medicamentos entre indivíduos com diabetes mellitus e hipertensão arterial no município do Rio de Janeiro, Brasil. Ciênc. saúde coletiva. 2014;19(6): 1673-1684.

14. Organização Mundial da Saúde (OMS). World Health Organization (WHO). Adherence to long-term therapies: evidence for action. Geneva: WHO, 2003.

15. Manfroi A, Oliveira FA. Dificuldades de adesão ao tratamento na hipertensão arterial sistêmica: considerações a partir de um estudo qualitativo em uma unidade de Atenção Primária à Saúde. Rev Bras de Med Fam e Com. 2006;2(7): 165-176.

16. Papaléo Netto M. Gerontologia: a velhice e o envelhecimento em visão globalizada. São Paulo: Atheneu, 2005.

17. Rocha $\mathrm{CH}$ et al. Adesão à prescrição médica em idosos de Porto Alegre, RS. Rev. Ciência \& Saúde Coletiva. 2008;13(Suppl): 703-710.

18. Gusmão JL, Mion Jr D. Adesão ao tratamento-conceitos. Rev Bras Hipertens. 2006;13(1): 23-25.

19. Leite SN, Guimarães MCL. Gestão da Assistência Farmacêutica. Módulo Transversal I. Universidade Federal de Santa Catarina. Florianópolis, 2011. Disponível em www.unasus.ufsc.br

20. Contradiopoulos AP. A avaliação na área da saúde: conceitos e métodos. In: Hartz ZM, editor. Avaliação em saúde: dos modelos conceituais à prática na análise da implantação de programas. Rio de Janeiro: Editora Fiocruz, 1997. p. 29-47

21. Minayo MCS, Sanches O. Qualitativo-quantitativo: oposição ou complementaridade? Cadernos de Saúde Pública. 1993;9(3): 239-248.

22. Matus C. Política, planejamento \& governo. Brasília: Ipea, 1996.

23. Matus C. O Plano como Aposta. São Paulo em perspectiva. 1991;5(4): 28-42.

24. Sala de Apoio à Gestão Estratégica. [Internet]. Brasília: Ministério da Saúde. Redes e Programas. Saúde Mais Perto de Você - Atenção Básica. Equipes de Saúde da Família. Disponível em: http://189.28.128.178/sage/

25. Conselho Nacional de Saúde. Resolução N 196 de 10 de outubro de 1996. Aprova as diretrizes e normas regulamentadoras de pesquisas envolvendo seres humanos. [cited 2011 Dez 10]. Available from: http://conselho.saude. gov.br/web_comissoes/conep/aquivos/resolucoes/23_out_versao_final_196_ENCEP2012.pdf.

26. Barreto JL, Guimarães MCL. Avaliação da gestão descentralizada da assistência farmacêutica básica em municípios baianos, Brasil. Cad. Saúde Pública. 2010;26(6): 1207-1220.

27. Santana RS et al. A institucionalização da seleção de medicamentos em hospitais públicos por meio do planejamento estratégico situacional. Rev. Adm. Pública. 2014;48(6): 1587-1603. 
28. Naves JOS, Silver LD. Evaluation of pharmaceutical assistance in public primary care in Brasilia, Brazil. Rev. Saúde Pública. 2005;39(2): 223-230.

29. Pereira LS et al. Clinical and epidemiological profile of elderly patients with chagas disease followed between $2005-2013$ by pharmaceutical care service in Ceará state, northeastern Brazil. Rev. Inst. Med. trop. 2015;57(2): 145-152.

30. Chemello $\mathrm{C}$ et al. Pharmaceutical care as a strategy to improve the safety and effectiveness of patients? pharmacotherapy at a pharmacy school: a practical proposal. Braz. J. Pharm. Sci.2014;50(1): 185-193.

\section{NOTA}

1 Trabalho de Conclusão realizado no Curso Especialização Gestão da Assistência Farmacêutica - EAD: Uma proposta em rede para a qualificação da assistência farmacêutica - Universidade Federal de Santa Catarina. 